\title{
METALS IN MICROFLUIDICS - COUPLING PLASMONICS, ELECTRON TRANSFER AND NANOFLUIDICS IN A MONOLITHIC STRUCTURE

\author{
S.P. Branagan ${ }^{l}$, N. Contento ${ }^{l}$, and P.W. Bohn ${ }^{1,2} *$
}

${ }^{1}$ Department of Chemical and Biomolecular Engineering, University of Notre Dame, Notre Dame, Indiana, USA

${ }^{2}$ Department of Chemistry and Biochemistry, University of Notre Dame, Notre Dame, Indiana, USA

\begin{abstract}
Present work details the characterization of engineered metallic features in several micro/nanofluidic structures. Three distinct analytical applications for these nanoporous structures are targeted: (1) molecular reactivity of surface-immobilized enzymes under confinement (2) plasmonically active sensing elements supporting enhanced optical transmission (3) fabrication of metallic structures for in situ monitoring of electron transfer reaction products. Two generally applicable Au/polymer structures are introduced, including $\mathrm{Au}$ nanocapillary array membranes (NCAMs) and $\mathrm{Au}$ embedded annular nanoband electrodes (EANEs).
\end{abstract}

\section{INTRODUCTION}

The ultimate goal of this program is to establish intelligent control of molecular transport in space and time at small length scales - enabling the construction of materials and structures that can sense molecular characteristics, e.g. size, charge, molecular shape, etc., then generate control signals that control transport based on those characteristics. Specifically, these experiments seek to manipulate, (separate, isolate, react, detect) low-mass samples with the same precision and level of control currently possible with bench-scale samples by combining microfluidic and nanofluidic structures under the rubric of integrated microfluidics.

These nanofluidically mediated interactions frequently require metallic elements in the flow path, thereby making it possible to realize plasmonic sensing, electrokinetic fluid switching and electrochemical transformations in a single integrated architecture. To effect these electrochemical transformations, a layer of $\mathrm{Au}$ is incorporated within the nanofluidic feature. Two of these structures are the Au nanocapillary array membrane (NCAM), and the $\mathrm{Au}$ embedded annular nanoband electrode (EANE). These NCAMs can be used to support plasmonic sensing via enhanced optical transmission, while maintaining compatibility with electrokinetic transport. EANEs, meanwhile, can be used to effect heterogeneous electron transfer events at very high efficiency inside the nanopore during electrokinetic pumping.

\section{MOLECULAR REACTIVITY UNDER CONFINEMENT}

A key practical reason for pursuing small scale architectures is the characteristic gain in reaction rate. Most enzymatic reactions in free solution are diffusion limited; this represents a transportimposed upper bound, regardless of the intrinsic capability of the enzyme species. Previously published work ${ }^{1}$ studied the behavior of enzymes geometrically-confined to the inside surface of a nanometer-scale pore. Horseradish peroxidase (HRP) catalyzes the oxidation of a fluorigenic substrate, Amplex Red (AR), to fluorescent resorufin in the presence of $\mathrm{H}_{2} \mathrm{O}_{2}$. Figure 1 depicts two configurations of surface- immobilized HRP, where the enzyme can be bound to either (A) a planar surface in a microchannel or (B) the interior of cylindrical nanopores as part of an integrated micro/nanofluidic structure. In both cases, the immobilized HRP

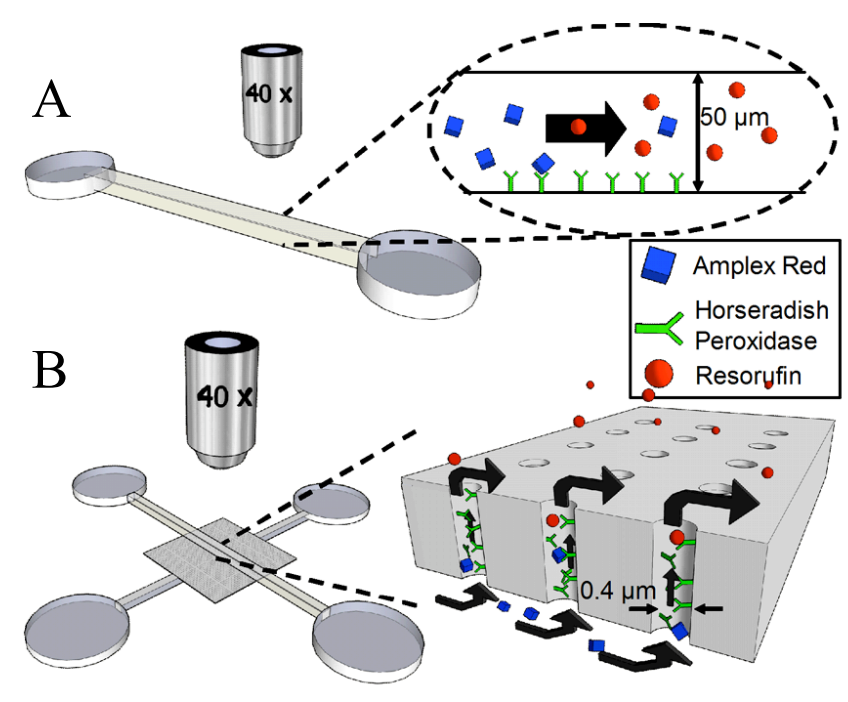

Figure 1: Schematics of planar-surface immobilized HRP reaction formats (A) single microchannel system, HRP immobilized on one planar surface (B) 3-dimensional micro/nanofluidic system with HRP immobilized on the interior nanopore walls. In all figures, chemical species are coded as: Amplex Red (blue) HRP (green), and Resorufin (red)

under confinement was characterized and compared to finite

element simulations of a modified Eley-Rideal mechanism to bracket the value of the initial reaction velocity. Initial reaction velocities were estimated to be $\geq 5$ times higher in microchannel, and $\geq 20$-fold higher in the nanopores than for the same enzyme in macroscale fluid solution (see Table 1).

Table 1: Comparison of enzyme-to-substrate ratios and initial rates for various enzyme reaction geometries

\begin{tabular}{|l|c|c|}
\hline & {$[\mathrm{E}] /[\mathrm{S}]$ ratio } & $\begin{array}{c}\text { Initial rate } / \mu \mathrm{M} \\
\mathrm{min}^{-1}\end{array}$ \\
\hline $\begin{array}{l}\text { Free solution } \\
\text { (literature) }^{2}\end{array}$ & $\sim 0.001$ & 1.8 \\
\hline $\begin{array}{l}\text { Planar surface in } \\
\text { microchannel }\end{array}$ & $\sim 0.001$ & 12 \\
\hline $\begin{array}{l}\text { Nanopore array } \\
\text { (experiment) }\end{array}$ & 100 & 36 \\
\hline $\begin{array}{l}\text { Nanopore array } \\
\text { (simulation) }\end{array}$ & - & $15-60$ \\
\hline
\end{tabular}
Hilton Head Island, South Carolina, June 6-10, 2010 
It is worth noting that the nanopore experiments exhibit a very different enzyme: substrate ratio $([\mathrm{E}] /[\mathrm{S}] \sim 100)$ compared to the same reaction in fluid solution $([\mathrm{E}] /[\mathrm{S}] \sim 0.001)$, however the total mass of enzyme molecules was similar. Therefore, we attribute the gain in reactivity to two factors, both related to confinement: (1) the high aspect ratio nanopore that constrains the diffusive boundary layer, greatly reducing the substrate-to-site diffusion time, and (2) the increase in [E/S] ratio in the femtoliter-size nanopore volume. These hypotheses as to the mechanistic origins of the improved reaction rate, however, require a more advanced detection strategy than ex situ fluorescence microscopy.

This challenge leads to one of the major motivations for pursuing metallic features in nanofluidic structures: the potential for electrochemical characterization in situ. The past decade has brought major advances in nanoelectrochemistry in parallel with improved fabrication techniques. $\mathrm{Au}$ is an attractive metal for incorporation into microfluidics primarily due to surface functionalization via the wide range of Au-thiol chemistries. Previous work by our group has pursued Au-functionalized nanopore membranes in a variety of configurations, three of which are shown in Figure 2.
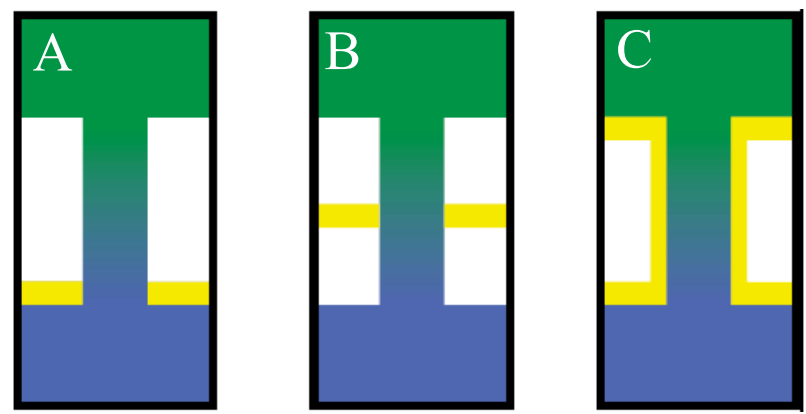

Figure 2: Au-polymer nanopore configurations (A) Au on one side of a nanocapillary array membrane (Au NCAM) (B) $A u$ contained within an embedded annular nanoband electrode (EANE) (C) fully covered Au membrane

The key electrical advantage of $\mathrm{Au}$, i.e. its excellent conductivity, also presents unique challenges for electrokinetic transport. Typically, the electrical current path electroosmosis ways through all-polymer nanofluidic devices will drive and/or electrophoresis through the solution, thereby constituting an effective means of driving nanofluidic transport. Incorporating a $\mathrm{Au}$ feature offers a parallel, low-resistance pathway which shorts out electrokinetic transport, often leading to unpredictable charge accumulation/depletion effects, and ultimately undesirable Faradaic reactions. Work by our group has probed these issues in detail, and has illuminated a number of strategies for successful injection across Au-functionalized nanopores of the types shown in Figure $2^{3,4}$. Injection traces for each of these geometries are shown in Figure 3.

\section{METALS IN MICROFLUIDICS SUPPORTING OPTOFLUIDIC SENSING}

Another important application of metals in microfluidic structures is plasmonic sensing of surface-bound analytes. Beyond the commercially available surface plasmon resonance biosensors, much work over the past decade has pursued enhanced optical transmission (EOT) through nanoporous metallic features ${ }^{5}, 6$. Previously published work detailed a new technique for mapping

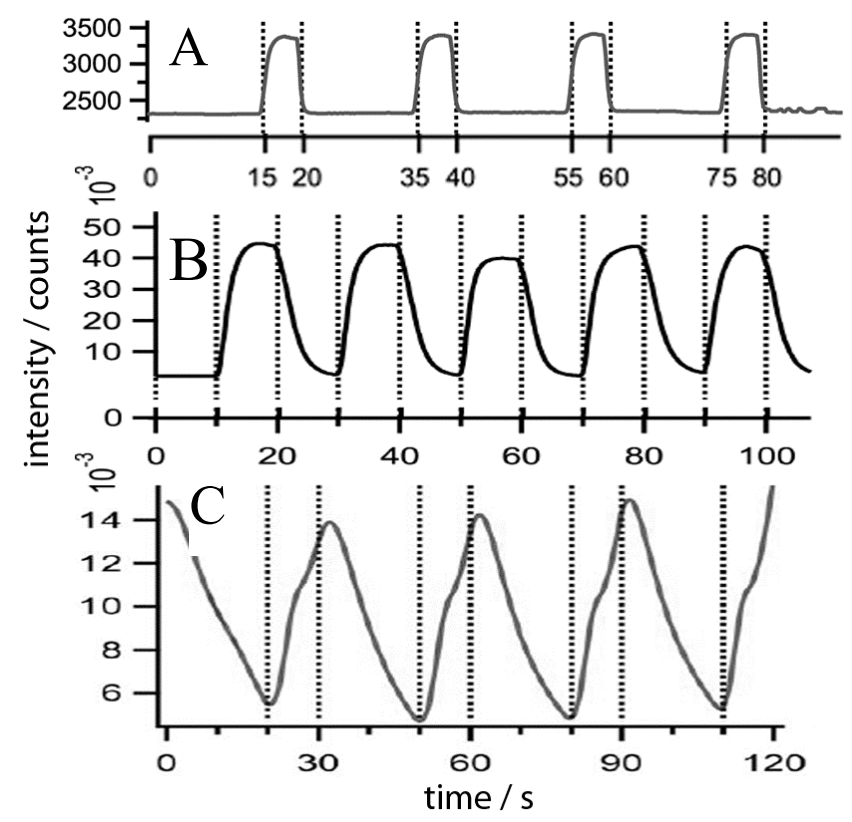

Figure 3: Injections across Au/polymer nanofluidic membranes (A) electrokinetic injections across a one-sided Au-polymer NCAM (B) electrokinetic injections across an EANE (C) pressure-driven flow across a fully coated Au/polymer membrane

enhanced optical transmission through integrated optofluidic nanopore arrays ${ }^{7}$. This technique, illustrated in Figure 4, involves monochromatic illumination and two microscope objectives. The first objective condenses the light to form a linearly polarized wavefront at the plane of the sample, at which the nanopore array is sensitive to both the wavelength, $\lambda$, and the angle (via the inplane wavevector component $\mathrm{k}_{\mathrm{x}}=(2 \pi / \lambda) \cdot \sin \theta$. The second objective, of higher numerical aperture (NA), collects the transmitted EOT image and maps the spectral information onto different coordinates on the CCD. Figure 4(B) shows just such an EOT image of the nanopore array, including the distinctive vertical bands of illumination, where different regions of the nanopore array transmit at varying efficiencies. During chemical sensing, these vertical bands shift with characteristic changes in the local refractive index, enabling label-free transduction of surface-bound chemical species. This technique is applicable to any of the published EOT-based detection formats ${ }^{8-10}$, and represents a much faster route to characterize the full spectrum of plasmonic resonant shifts.

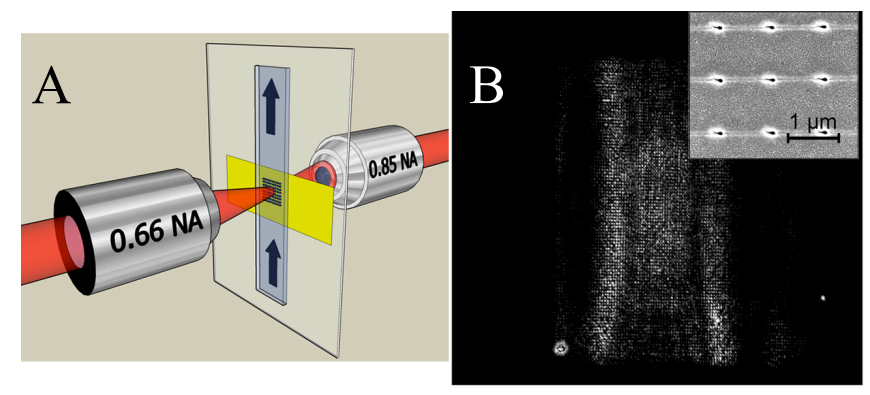

Figure 4: Wavevector resolved spectroscopic imaging of enhanced optical transmission (EOT) (A) schematic of the optical technique with nanopore-array and microchannel in the sample plane of the EOT microscope (B) EOT image of a $100 \times 100 \mu m$ pore array, with corresponding SEM close up (inset) 


\section{IN SITU NANOELECTROCHEMISTRY FOR ELECTRON TRANSFER REACTIONS}

As discussed in Section II, nanoelectrochemistry offers one important strategy for reaction monitoring in situ. Current work pursues a novel micro/nanofluidic structure (Figure 5). This structure is fabricated out of glass (top microchannels) while the bottom nanochannels are fabricated in hard polydimethylsiloxane elastomer (h-PDMS) ${ }^{11}$. This structure supports simultaneous electrochemical and fluorescence monitoring of nanopore reactors. Current work pursues vanillyl-alcohol oxidase and D-amino acid oxidase families of enzyme pairs, and sarcosine oxidase (SO) specifically. Here, the SO incorporates a covalently-bound $\mathrm{FAD} / \mathrm{FADH}_{2}$ cofactor, which cycles between active and inactive states which can be followed through the cofactor (FAD) fluorescence, while the product generation is monitored electrochemically $\left(\mathrm{H}_{2} \mathrm{O}_{2}\right)$. Since many different oxidase/oxidoreductase enzymes share these common characteristics, this structure enables access to a wide range of enzymatic reactions $^{12}$.

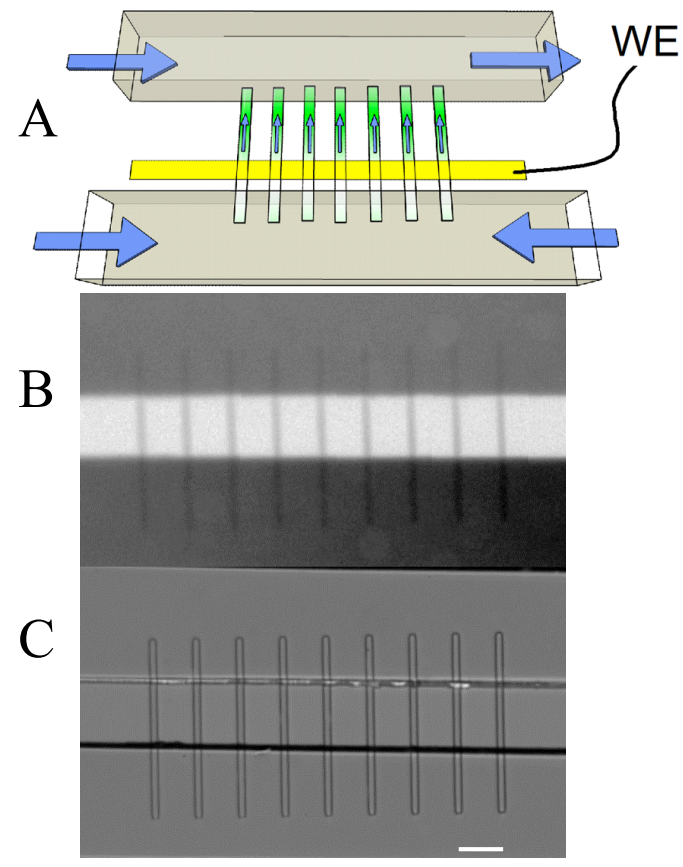

Figure 5: Nanochannel reactors coupled to an in situ working electrode (A) schematic showing location of working electrode and flow paths (B) fluorescence micrograph of nanochannel reactors (no electrode) (C) brightfield micrograph of the same structure.

\section{CONCLUSIONS}

Integrating metallic features into three-dimensional microfluidic-nanofluidic architectures offers one approach to many of the most interesting challenges in integrated microfluidics. Direct transduction of chemical reactions near an EANE, for example, provides the most direct measurement yet of nanoscale electron transfer reaction in situ. Similarly, the fundamental reactivity of enzyme species may be examined through simultaneous electrochemical and optical detection. Lastly, labelfree sensing via optofluidic coupled EOT enables an attractive route for transduction of chemical products within subwavelength nanoporous structures. These on-chip analysis techniques represent an advancement towards the next generation of smallscale, lab-on-a-chip style analytical devices.

\section{ACKNOWLEDGEMENTS}

This work was supported by the Department of Energy through grant DE FG02 07ER15851, and by the Army Corps of Engineers through contracts W9132T-07-2-0003 and W9132T-102-0010.

\section{REFERENCES}

[1] Z. Wang, T. L. King, S. P. Branagan and P. W. Bohn, Analyst, 2009, 134, 851-859.

[2] G. H. Seong, J. Heo and R. M. Crooks, Anal Chem, 2003, 75, 3161-3167.

[3] A. Piruska, S. Branagan, D. M. Cropek, J. V. Sweedler and P. W. Bohn, Lab on a Chip, 2008, 8, 1625-1631.

[4] A. Piruska, S. P. Branagan, A. Minnis, Z. Wang, D. M. Cropek, J. V. Sweedler and P. W. Bohn, Lab on a Chip, 2010, DOI: $10.1039 / b 924164 g$.

[5] T. W. Ebbesen, H. J. Lezec, H. F. Ghaemi, T. Thio and P. A. Wolff, Nature, 1998, 391, 667-669.

[6] H. F. Ghaemi, T. Thio, D. E. Grupp, T. W. Ebbesen and H. J. Lezec, Phys Rev B, 1998, 58, 6779-6782.

[7] S. P. Branagan and P. W. Bohn, Opt Express, 2009, 17, 18995-19005.

[8] J. M. Yao, M. E. Stewart, J. Maria, T. W. Lee, S. K. Gray, J. A. Rogers and R. G. Nuzzo, Angew Chem Int Edit, 2008, 47, 5013-5017.

[9] N. H. Mack, J. W. Wackerly, V. Malyarchuk, J. A. Rogers, J. S. Moore and R. G. Nuzzo, Nano Lett, 2007, 7, 733-737.

[10] A. De Leebeeck, L. K. S. Kumar, V. de Lange, D. Sinton, R. Gordon and A. G. Brolo, Anal Chem, 2007, 79, 4094-4100.

[11] H. Schmid and B. Michel, Macromolecules, 2000, 33, 30423049.

[12] D. P. H. M. Heuts, N. S. Scrutton, W. S. McIntire and M. W. Fraaije, Febs J, 2009, 276, 3405-3427.

\section{CONTACT}

*P.W. Bohn, tel: +1-574-631-1835; pbohn@nd.edu 